\title{
Evaluation of PE Films Having NIR-Reflective Additives for Greenhouse Applications in Arid Regions
}

\author{
Syed K. H. Gulrez, ${ }^{1}$ Ahmed M. Abdel-Ghany, ${ }^{2}$ Ibrahim M. Al-Helal, ${ }^{2}$ Saeed M. Al-Zaharani, \\ and Abdullah A. Alsadon ${ }^{3}$ \\ ${ }^{1}$ Chemical Engineering Department, College of Engineering, King Saud University, Riyadh 11451, Saudi Arabia \\ ${ }^{2}$ Agricultural Engineering Department, College of Food and Agricultural Sciences, King Saud University, P.O. Box 2460, \\ Riyadh 11451, Saudi Arabia \\ ${ }^{3}$ Plant Production Department, College of Food and Agricultural Sciences, King Saud University, Riyadh 11451, Saudi Arabia
}

Correspondence should be addressed to Syed K. H. Gulrez; sgulrez@ksu.edu.sa

Received 25 November 2012; Revised 14 January 2013; Accepted 14 January 2013

Academic Editor: Amit Bandyopadhyay

Copyright (C) 2013 Syed K. H. Gulrez et al. This is an open access article distributed under the Creative Commons Attribution License, which permits unrestricted use, distribution, and reproduction in any medium, provided the original work is properly cited.

\begin{abstract}
Linear-low-density-polyethylene- (LLDPE-) based formulations with several near-infrared- (NIR-) reflective pigments were prepared by melt blending technique and their subsequent films were prepared by blown film extrusion process. Thermal properties of these films were evaluated using differential scanning calorimetry (DSC). The results showed that the melting and crystallization temperatures ( $T_{m}$ and $T_{c}$, resp.) of these formulations were almost similar to that of control resin. The melt viscosity was measured by stress-controlled rotational rheometer and melt flow index (MFI) instruments. Rheological measurements indicated that the blend formulations with NIR-reflective additive have similar melt viscoelastic behavior (storage modulus and dynamic viscosity) to the control resin. The mechanical test performed on NIR-reflective films showed similar values of tensile strength for blend samples as that of control resin. The spectral radiometric properties of the blend films were evaluated in the solar wavelength range of 200-1100 nm and found to be improved over the control sample without having NIR-reflective pigment.
\end{abstract}

\section{Introduction}

Climate in arid regions is characterized by hot, dusty, dry, and long summer. The maximum ambient temperature in these regions is usually well above $45^{\circ} \mathrm{C}$, solar radiation flux is quite high (up to $1200 \mathrm{Wm}^{-2}$ ), and the relative humidity is as low as $10 \%$. Moreover, the water resources are scarce and brackish [1]. Such harsh weather conditions negatively affect the overall production of the crops. One of the most common ways to protect the crops from excessive solar radiation is to use the greenhouse coverings [2]. They protect crops from adverse weather conditions, such as rain, wind, heat burn, insects, and diseases [3]. Nevertheless, the harsh weather conditions also affect the shelf life of greenhouse covering material and rapidly degrade their optical and mechanical properties. Some of the practical methods adopted to overcome the heat buildup inside greenhouse are ventilation and evaporative cooling systems [4]. Ventilation can suitably be adopted in the regions where ambient temperature is around $30^{\circ} \mathrm{C}$. However, in the extreme summer conditions, it is uncommon to use a ventilation method alone, since it replaces the overheated greenhouse air with a very hot ambient air and cannot provide adequate cooling [5-8]. Evaporative cooling (wet pad-fan system or fogging system) is alternatively used to provide cool and moist air for plant growth during summers. The most essential requirement for such system to operate efficiently in an arid climate is the availability of pure and fresh water resource that can be used for continuous wetting of the pad and pumping it through the nozzles of a fogging system. However, in arid regions such as Saudi Arabia, the water resources are saline and brackish. The use of such saline water causes fast deterioration in the cooling performance of the wet pad-fan systems. The clogging by the salt buildup on the pad surfaces restricts the air flow leading to an increased electrical energy consumption as well as temperature rise inside the greenhouse $[9,10]$. For these 
reasons, ventilation and evaporative cooling techniques are not practically suitable to adopt in this region. A greenhouse covering that can reduce the solar irradiative load on crops by preventing a portion of unwanted incident radiation would be ideal for agriculture applications in arid regions.

Among the choice of greenhouse covering materials, plastics are more suitable than glass principally for their cheapness, lightness, and large size features [11]. Mechanical properties of a greenhouse covering film are quite an important factor, as it undergoes severe weathering conditions. The film's response to stresses (mainly due to thermal variations and weathering) evolves with time since the polymeric materials degrade under ultraviolet radiation, heat, and microorganism [12]. Due to their low cost and availability, polyethylene- (PE-) based monolayer films are quite popular in the greenhouse covering application. PE films with the thickness of about $140-200 \mu \mathrm{m}$ are often characterized by a shelf life of as long as 2-3 years [1, 13].

The optical properties of chosen plastic films are vital as well, as they largely govern the crop yield. Solar radiation induces photosynthesis, which is essential for the plant growth and provides energy to the greenhouse system [14]. The spectral distribution of solar radiation reaching earth surface has about $40 \%$ of the total energy emitted in the nearinfrared (NIR) radiation range $(700-2500 \mathrm{~nm})$ and about $39 \%$ in the photosynthetically active radiation (PAR) range $(400-700 \mathrm{~nm})[11,15]$. A provision that can allow the PARs to reach the crops and inhibit NIR radiations (i.e., $50 \%$ of global solar radiation) would be ideal for the agriculture production in arid regions. This can be accomplished by using a radiation-filtering greenhouse cover that can reflect the NIR radiations and transmit selectively the PARs to the crops.

PE-based films and sheets with several NIR-reflective additives have been prepared earlier and reported in the literature $[16,17]$. These films are found to reduce the solar radiation across the whole solar spectrum including the PAR region. The PAR transmittance for these films was found to be in the range from $62 \%$ to $72 \%$, while their NIR reflectance was in the range from $37 \%$ to $54 \%[16,17]$. However, the presence of NIR-reflective additives adversely affected the lifetime of covering films thus making them unsuitable for the greenhouse application. Verlodt and Verschaeren have reported the development of a novel NIR-reflective film having stronger reflection of NIR capacity combined with a higher PAR transmission [18]. The performance of this new film was evaluated against a standard PE film for its application as a greenhouse cover. Results showed that the PAR transmittance of the developed film was quite good (almost the same as that of the standard PE film). However, its efficiency to reflect NIR radiation was quite low (though $4.3 \%$ higher than that of the standard PE film). López-Marín et al. have also examined the effectiveness of a PE film having NIRreflective pigment as a greenhouse cover [19]. Their results also showed that the use of NIR-reflective additive caused a shift in the whole spectrum to a lower transmission side resulting around $15-20 \%$ loss in the PAR transmission as compared to the standard PE cover. Impron et al. studied two PE films incorporating NIR-reflective pigments with varying concentrations [20]. The measured PAR transmittance for
TABLE 1: Important physical parameters of polymer resins used in this study.

\begin{tabular}{lccccc}
\hline $\begin{array}{l}\text { Sr. } \\
\text { no. }\end{array}$ & Sample & $\begin{array}{c}\text { Density } \\
(\mathrm{gm} / \mathrm{cc})\end{array}$ & $\begin{array}{c}\text { MFI } \\
(\mathrm{g} / 10 \mathrm{~min})\end{array}$ & $\begin{array}{c}\text { Strength } \\
(\mathrm{MPa})\end{array}$ & $\begin{array}{c}\text { Elongation } \\
(\%)\end{array}$ \\
\hline 1 & LLDPE 6821 & 0.921 & 0.80 & 13 & 730 \\
2 & EVA 218 & 0.941 & 1.7 & 39 & 500 \\
\hline
\end{tabular}

such films was about $77-80 \%$, while NIR reflectance was about $21-26 \%$. Mutwiwa et al. studied the effects of coating the PE roof of a naturally ventilated greenhouse with NIRreflective pigment on the greenhouse microclimate [21]. According to their results, the PAR transmittance of the coated film was estimated to be around $72 \%$, which is quite encouraging. However, their NIR reflectance was estimated to be merely $22 \%$. The coating of NIR-reflective pigment, however, has its own limitations to be practically used for greenhouse covering applications.

Based on studies reported in the literature, it can be stated that the development of PE films with NIR-reflecting properties for greenhouse covering application is still under the developmental stage. The objective of this project was to satisfy the need for a new generation of greenhouse covering materials with a good shelf life (2-3 years) that can reflect the NIR portion of the solar spectrum, yet allowing the PARs that are essential for plant growth to pass through. In this study, PE-based formulations using different NIRreflective pigments were prepared by the blown film extrusion technique. These films were characterized thoroughly to investigate the influence of additives on the film processing and its mechanical properties. The radiometric properties of these films were also evaluated in terms of NIR reflectance and PAR transmittance to study the influence of these pigments on the spectral irradiative behavior of the film.

\section{Materials and Methods}

A film grade LLDPE resin was selected from a local polyolefin producing industry (SABIC, Saudi Arabia). Ethylene-vinyl acetate polymer resin used in this study was obtained from ExxonMobil Chemicals, USA (Escorene Ultra FL 00218). Some of the important physical parameters for these raw materials used in preparing blend formulations are listed in Table 1.

Various NIR-reflective additives used in the present work were supplied by Shepherd, Cincinnati, USA. Table 2 shows their compositions and other important properties such as total solar reflectance (TSR) and heat buildup (HBU). Total solar reflectance is the percentage of the total solar energy reflected by the pigment. Heat buildup is the temperature raised due to solar radiations being absorbed by the pigment and is measured according to ASTM D4803 method.

Commercially available UV additives such as antioxidants and UV stabilizers used in this study were obtained from their major suppliers, and their description is listed in Table 3. These additives were used in different concentrations and combinations. 
TABLE 2: Composition and important properties of NIR-reflective additive used in this study.

\begin{tabular}{lcccc}
\hline Sample no. & Pigment code & Composition & TSR $^{*}$ & HBU $^{*}$ \\
\hline NIR1 & Blue 424 & CoAl & $42 \%$ & $31^{\circ} \mathrm{C}$ \\
NIR2 & Yellow 10P110 & NiSbTi & $69 \%$ & $20^{\circ} \mathrm{C}$ \\
NIR3 & Orange 10P225 & CrSbTi & $63 \%$ & $22^{\circ} \mathrm{C}$ \\
NIR4 & Green 223 & CoNiZnTi & $25 \%$ & $35^{\circ} \mathrm{C}$ \\
NIR5 & Brown 10P850 & MnSbTi & $35 \%$ & $26^{\circ} \mathrm{C}$ \\
\hline
\end{tabular}

\section{Experimental}

3.1. Compounding. A blend of LLDPE 6821 (80\%) and EVA $218(20 \%)$ was prepared and used as a base resin (control sample) to prepare all other formulations reported in this study. Composition of control material and different blends prepared are listed in Table 4. The control resin (S1) was dry-blended with different additives (NIR reflective, UV stabilizer, antioxidants, etc.) in different ratios. The dry-blend was pelletized using an intermeshing and corotating twin screw extruder, Farrell FTX20 (screw dia $26 \mathrm{~mm} ; / d$ ratio 35 ). The screw has got both the dispersive and distributive mixing elements. The dry-blends were fed to the extruder that was operating at a screw speed of $12 \mathrm{rpm}$ and an average processing temperature of $235^{\circ} \mathrm{C}$. The melt pressure was about 7 bars. The extrudate was cooled in a water bath, dried, and pelletized for further use.

3.2. Blown Film Preparation. Thin PE films of various thicknesses $(60-200 \mu \mathrm{m})$ were prepared by blown film extrusion techniques, using the above-mentioned formulations (Table 4). Films were drawn at $30^{\circ} \mathrm{C}$ through a ribbon die by a takeoff machine (model Shouman FPE200, Egypt) at a varying speed of $0.5-2 \mathrm{~m} / \mathrm{min}$ in order to obtain the films of desired thickness. The temperature was controlled with a water-cooled thermostatic system connected with the takeoff machine.

3.3. Differential Scanning Calorimetry. Differential scanning calorimetry (Shimadzu DSC-60) was used for the thermal analysis of control and blend resins. Specimens of about 6$7 \mathrm{mg}$ each were prepared by "shaving off" a thin layer $(<1 \mathrm{~mm}$ thickness) of the pellets to minimize the thickness effect. The specimen was placed in an aluminum pan without being sealed then placed in the DSC oven in air at an atmospheric pressure. Samples were heated at $5^{\circ} \mathrm{C} / \mathrm{min}$ from room temperature to $200^{\circ} \mathrm{C}$, held there for $10 \mathrm{~min}$, and then cooled back to room temperature at the same rate as of heating. The peak temperature of the melting curve was recorded as the melting temperature $\left(T_{m}\right)$ of these materials. The onset crystallization temperature $\left(T_{c}\right)$ was determined at the beginning of the crystallization peak (at the intersection of the peak slope with the baseline). The melting and crystallization enthalpies, $\Delta H_{m}$ and $\Delta H_{c}$, were determined from the corresponding peak areas in the heating and cooling DSC scans, respectively.

3.4. Melt Flow Index. The melt flow rates of the samples were determined by using a Dynisco Polymer Test Melt Indexer,
USA, at $190 \pm 1.0^{\circ} \mathrm{C}$ using a $2.16 \mathrm{~kg}$ load and a dwell time of 300 seconds. ASTM 1238 was used as the guideline for this procedure.

3.5. Viscoelastic Properties. The viscoelastic properties of control and blend resins were characterized using ARG2 rheometer (TA instruments, USA). The samples were compression molded at $190^{\circ} \mathrm{C}$ under pressure with required diameter disks to fit the rheometer circular plates. The linear viscoelastic functions were measured using the parallel plate geometry (diameter of $25 \mathrm{~mm}$ and gap of 1000 micron). Frequency sweep was performed between 0.1 to $100 \mathrm{rad} / \mathrm{s}$ at $190^{\circ} \mathrm{C}$. Strain sweeps were performed priori to ensure that the frequency sweep tests were done in the linear viscoelastic region. Additionally, time sweeps were also performed to make sure that the polymer is stable (e.g., no degradation is taking place during the length of measurement). The temperature was stable within $0.5^{\circ} \mathrm{C}$ over the range used in this study. All tests were performed in the atmospheric air.

3.6. Tensile Test. The tensile properties of the film specimens were measured according to ASTM D882 using a Hounsfield H100 KS series tensile testing machine. The samples were conditioned prior to testing at room temperature for 24 hours. The tensile tests were performed at a crosshead speed of $20 \mathrm{inch} / \mathrm{min}$. The reported measurements for all of the above tests represent the median of three experiments.

3.7. Radiometric Properties. The spectral transmittance and reflectance of the samples were measured at normal incidence using Black-Comet (StellarNet Inc., USA) spectrophotometer, scanning between 200 and $1100 \mathrm{~nm}$ at $0.5 \mathrm{~nm}$ intervals in the UV-Vis range $(200-750 \mathrm{~nm})$ and at $1 \mathrm{~nm}$ intervals in the NIR range $(750-1100 \mathrm{~nm})$. The measured data were averaged at each $20 \mathrm{~nm}$ interval.

\section{Results and Discussion}

4.1. DSC Analysis. Figure 1 shows the dynamic thermogram (heating and cooling) of control sample (S1) and blend composites having NIR-reflective additives (S2 to S6). Figure 2 depicts the thermograms of control (S1) and the blend composites having mixture of various NIR and other UV additives (S1 and S7 to S9). The thermograms were shifted vertically for the ease of the presentation. The DSC data including melting and crystallization temperature $\left(T_{m}\right.$ and $T_{c}$, resp.) are summarized in Table 5.

The endothermic melting peaks of these samples appear to be quite similar. There was no significant change in the $T_{m}$ of these formulations compared with the control resin (S1). Therefore, it can be said that the incorporation of these additives (NIR reflective, UV absorber, antioxidants, etc.) does not significantly affect the melting characteristics of the neat polymer blend. Likewise, the shapes of the exothermic crystallization peak for control and blend samples were also quite similar to each other. However, deviation was found in the $T_{c}$ which is slightly higher for the blend samples than 
TABLE 3: List of UV additives used in this study.

\begin{tabular}{lll}
\hline Sample no. & Functionality & Description \\
\hline UVA1 & UV stabilizer masterbatch & $\begin{array}{l}\text { It is a concentrate of light stabilizers and thermal stabilizers in pellet form. It contains UV } \\
\text { stabilizers and IR thermal stabilizers in polyethylene carrier. } \\
\text { It is a concentrate of antioxidant agents in pellet form. It contains a combination of } \\
\text { antioxidants in polyethylene carrier. }\end{array}$ \\
UVA3 & Antioxidant masterbatch & $\begin{array}{l}\text { It is a UV stabilizer specifically developed for polyolefins. It is suitable for all applications } \\
\text { using LDPE, LLDPE, and PP. }\end{array}$ \\
\hline
\end{tabular}

TABLE 4: List of the samples prepared and used this study.

\begin{tabular}{lc}
\hline Sample no. & Composition \\
\hline S1 & LLDPE 6821 (80\%)+ EVA 218 (20\%) \\
S2 & S1 $(99.5 \%)+$ NIR1 $(0.5 \%)$ \\
S3 & S1 $(99.5 \%)+$ NIR2 $(0.5 \%)$ \\
S4 & S1 $(99.5 \%)+$ NIR3 $(0.5 \%)$ \\
S5 & S1 $(99.5 \%)+$ NIR4 $(0.5 \%)$ \\
S6 & S1 $(99.5 \%)+$ NIR5 $(0.5 \%)$ \\
S7 & S1 $(98 \%)+$ UVA1 $(2 \%)$ \\
S8 & S3 $(99.5 \%)+$ UVA2 $(0.25 \%)+$ UVA3 $(0.25 \%)$ \\
S9 & S5 $(99.5 \%)+$ UVA2 $(0.25 \%)+$ UVA3 $(0.25 \%)$ \\
\hline
\end{tabular}

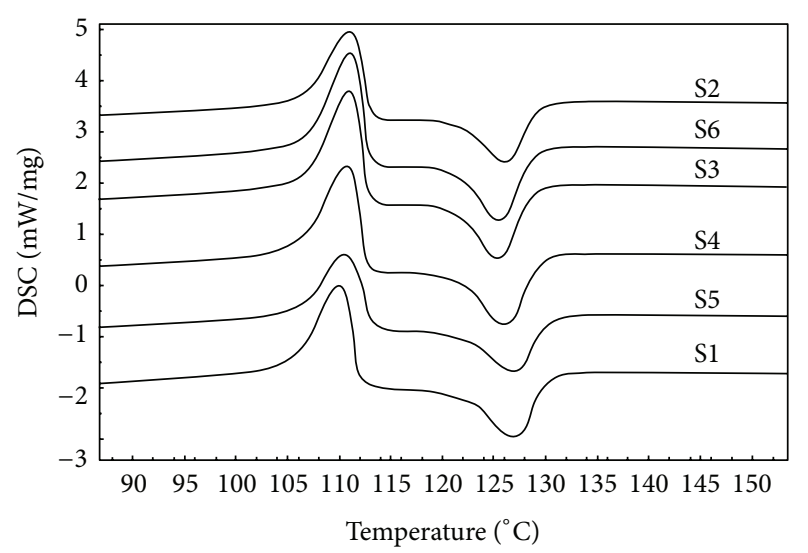

FIGURE 1: Heating and cooling thermograms of control and blend samples with NIR-reflective pigments.

control (S1). This finding might be attributed to the heterogeneous nucleating effect induced by the presence of additives (NIR-reflective pigments, UV absorber, antioxidants, etc.) in blend samples [22].

4.2. Tensile Properties. The tensile data for control and blend samples is shown in Table 6. Tensile strength of blend samples is usually lower than the control resin (except in the case of S2 and S7). This indicates that there is no orientation of the polymer chains as a result of introducing various NIR and UV additives in control resin, and, hence, the mechanical strength of blend samples is reduced [23]. Percent deformation at the break of blend samples is also shown to be decreased by the incorporation of these NIR and UV additives since they restrict the motion of the polymer chains. The additives might

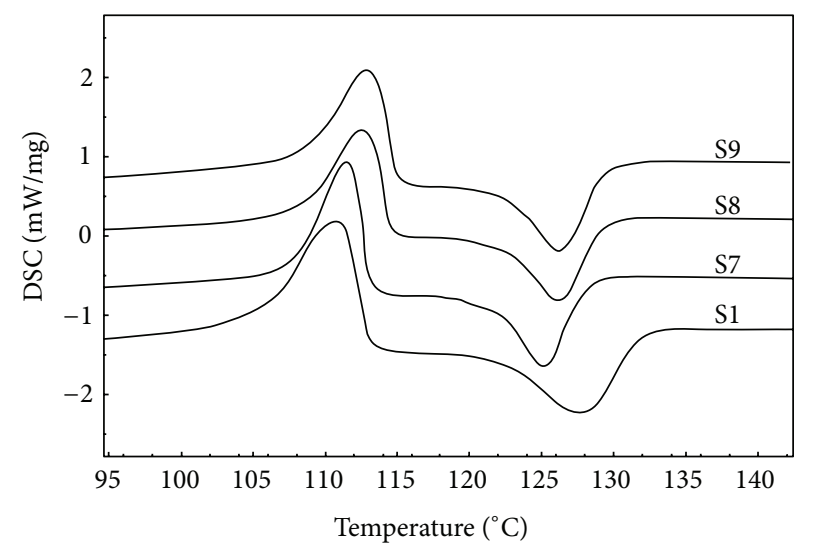

FIgURE 2: Heating and cooling thermograms of control and blend samples with NIR reflective and other UV additives.

TABLE 5: Thermodynamic properties of control resin and blend samples.

\begin{tabular}{lcccc}
\hline Sample & $T_{c}\left({ }^{\circ} \mathrm{C}\right)$ & $\Delta H_{c}(\mathrm{~J} / \mathrm{g})$ & $T_{m}\left({ }^{\circ} \mathrm{C}\right)$ & $\Delta H_{m}(\mathrm{~J} / \mathrm{g})$ \\
\hline S1 & 109.82 & 46.17 & 127.54 & 33.40 \\
S2 & 110.86 & 36.64 & 125.95 & 27.75 \\
S3 & 110.82 & 45.42 & 125.13 & 33.87 \\
S4 & 110.65 & 44.29 & 125.89 & 37.13 \\
S5 & 110.53 & 35.67 & 126.83 & 28.62 \\
S6 & 110.94 & 46.52 & 125.37 & 36.58 \\
S7 & 111.50 & 28.35 & 125.36 & 27.72 \\
S8 & 111.46 & 34.12 & 125.01 & 22.52 \\
S9 & 112.82 & 31.16 & 126.19 & 24.08 \\
\hline
\end{tabular}

reduce the flexibility by perturbing the chain motion of the control resin [24].

4.3. MFI and Rheological Properties. The MFI is a simple, easily obtainable viscosity parameter that indicates the physical properties and molecular structure of the polymer [25]. The effect of the incorporation of additives on the melt flow of control and blend samples is shown in Table 6. It is evident that there is no significant change in the melt flow index of these blend samples as compared to the control. This confirms that the blend formulations have similar flow characteristic and processing profile as the control resin [26].

The analysis of the viscoelasticity at lower frequency range can provide information about the microstructure of the final blend morphology that would be helpful to 


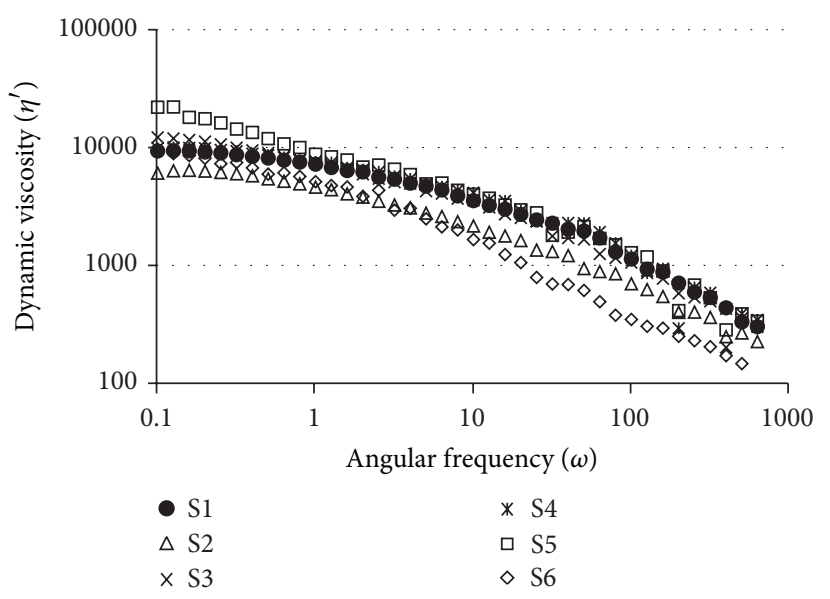

(a)

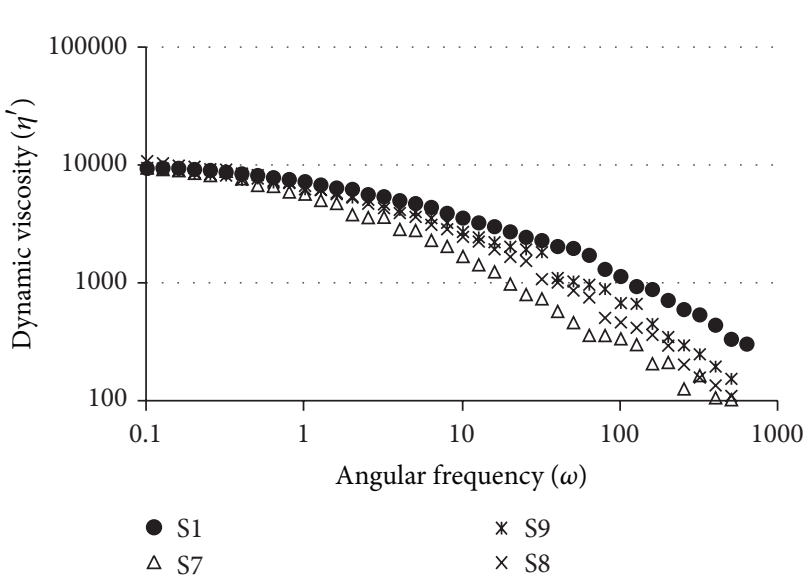

(b)

FIGURE 3: Frequency sweep rheogram for (a) control and blend samples with NIR-reflective pigments and (b) control with NIR reflective and other UV additives.

TABLE 6: MFI and tensile data for control and various blend samples.

\begin{tabular}{lccc}
\hline Sample no. & $\begin{array}{c}\text { MFI } \\
(\mathrm{g} / 10 \mathrm{~min})\end{array}$ & $\begin{array}{c}\text { Tensile strength } \\
(\mathrm{MPa})\end{array}$ & $\begin{array}{c}\text { Elongation } \\
(\%)\end{array}$ \\
\hline S1 & 0.824 & 13.11 & 870 \\
S2 & 0.862 & 14.75 & 716 \\
S3 & 0.801 & 12.09 & 852 \\
S4 & 0.812 & 11.25 & 690 \\
S5 & 0.823 & 12.75 & 836 \\
S6 & 0.781 & 12.74 & 600 \\
S7 & 0.821 & 14.09 & 644 \\
S8 & 0.790 & 12.01 & 606 \\
S9 & 0.836 & 12.71 & 620 \\
\hline
\end{tabular}

evaluate the interfacial interaction between the different phases of polymer blends. At low frequency, the effect of the flow-induced molecular orientation on viscoelasticity becomes more or less negligible and is mainly governed by the polymeric microstructure rather than the flow-induced molecular artifacts [27]. Frequency sweep profiles (plot of the dynamic viscosity $\left(\eta^{\prime}\right)$ versus the angular frequency $(\omega)$ ) for the control sample (S1) and blends having NIR-reflective additives (S2 to S6) are shown in Figure 3(a). Figure 3(b) depicts the frequency sweep profiles for blend samples having NIR and other UV additives (S7 to S9).

The effect of oscillation frequency on the dynamic viscosity of these samples measured at $190^{\circ} \mathrm{C}$ shows that as the frequency increases, the viscosity decreases due to the increased number of polymeric chain entanglements being broken than the number of new entanglements being reformed [26-28]. The $\eta^{\prime}$ of these various blend samples is more or less similar to that of control which is an indicative of the fact that these blend samples are rheologically similar to the control one. However, it is interesting to note that loss in viscosity $\left(\eta^{\prime}\right)$ with increased angular frequency $(\omega)$ is more prominent for the samples having NIR and other UV additives.

The storage modulus $\left(G^{\prime}\right)$ of control (S1) and blend resins with NIR-reflective additives (S2 to S6) are plotted against the loss modulus $\left(G^{\prime \prime}\right)$ as shown in Figure 4(a), while similar plot for blends having NIR and other UV additives is shown in Figure 4(b).

It can be observed that the slopes of the curves for blend samples are slightly shifted towards the $y$-axis. It manifests that blend samples exhibit better elastic properties than control. This might be due to the nature of additives (NIR reflective, UV stabilizer, antioxidants, etc.) that are contributing to the elasticity of polymeric microstructure.

4.4. Radiometric Properties. The solar irradiative characteristics of the prepared films were measured at normal incidence in the wavelength range of $200-1100 \mathrm{~nm}$ and illustrated in Figures 5(a) and 5(b). Figure 5(a) shows the reflectance spectra for control (S1) and blend samples having NIRreflective pigments (S2 to S6), while Figure 5(b) shows the same for blend samples containing NIR-reflective pigment and other UV stabilizers. The transmittance profile for these two sets of samples is shown in Figures 6(a) and 6(b), respectively. Average transmittance and reflectance values in various spectral regions (UV, PAR, and NIR) are summarized in Table 7.

From the results, it is evident that the blend film samples show an improvement in NIR reflectance as compared to the control sample S1. It is observed that S9 (having combination of NIR-reflective pigment and UV additives) shows the highest reflective capacity as compared to other blend samples. Those blend samples which contain additional UV stabilizers (S7 to S9) show a relatively lower transmittance values in UV region. The PAR transparency (an essential requirement for plant growth) is sufficiently high for control and most of blend samples (except S6 and S9). These results are an indicative of the right selection of the NIR-reflective pigments, 


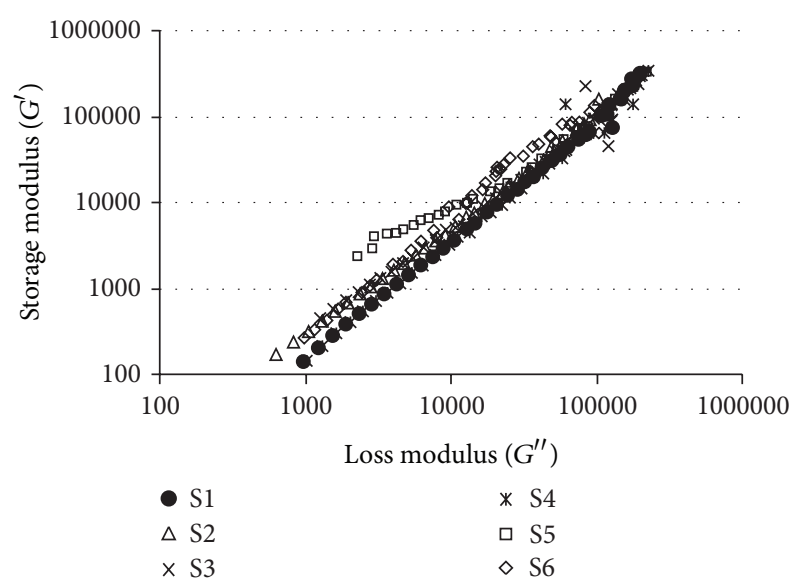

(a)

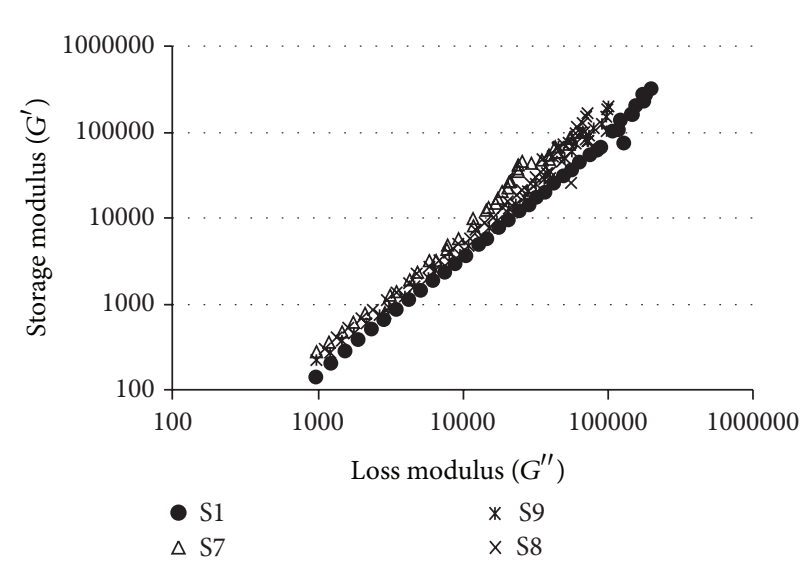

(b)

Figure 4: $G^{\prime}$ versus $G^{\prime \prime}$ curve for (a) control and blend samples with NIR-reflective pigments and (b) control with NIR reflective and other UV additives.

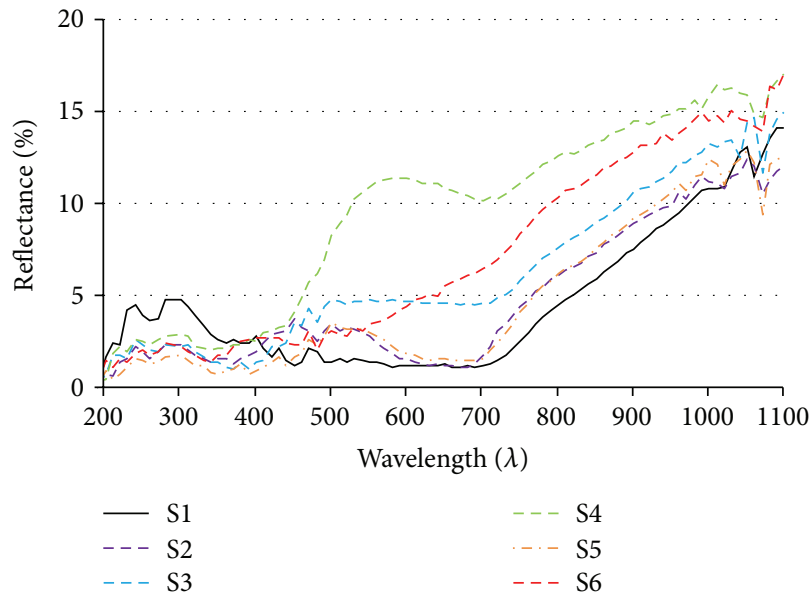

(a)

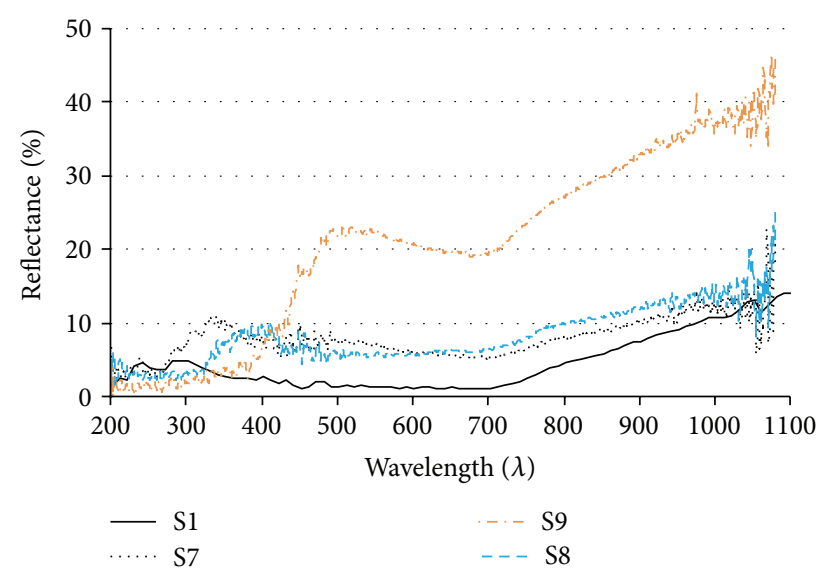

(b)

FIGURE 5: Spectral reflectance of (a) control and blend samples with NIR-reflective pigments and (b) control with NIR reflective and other UV additives.

and the formulations prepared thereof can be potentially tested for their use as a prototype greenhouse covering agent. However, these studies are still in the primitive stage since the reflectance properties of these films did not reach the ultimate goal. There is still a need to enhance the NIR reflectance of these formulations. Further work in this direction is currently under progress to improve the reflectance (by varying the loading ratio of NIR-reflective pigments, using different types of NIR-reflective pigments with higher TSR values, etc.).

\section{Conclusion}

A series of blend formulations has been prepared and tested for their suitability as a greenhouse covering film in this study. The polymer blends were successfully prepared by melt blending technique using several additives (NIR reflective, UV stabilizer, antioxidant, etc.), and their subsequent films with specific thickness were prepared by blown film extrusion technique. The DSC analysis and MFI data revealed that the thermal and flow characteristics of new formulations having NIR and UV additives are not very different from control, which is a positive attribute for the cost-effective processing of these formulations. The rheological characterization of these blends showed that their viscoelastic properties are quite similar to that of the control. However, the incorporation of various additives in control resin resulted in increased elastic modulus. The measurement of mechanical properties showed that the tensile strength of blend samples was not greatly affected by the incorporation of these additives, and these films can withstand the aggressive environment during their use as greenhouse covering films in arid region. The radiometric properties were found to be improved for these formulations. However, there is a need to further improve the NIR reflectance of the current formulations. To achieve 


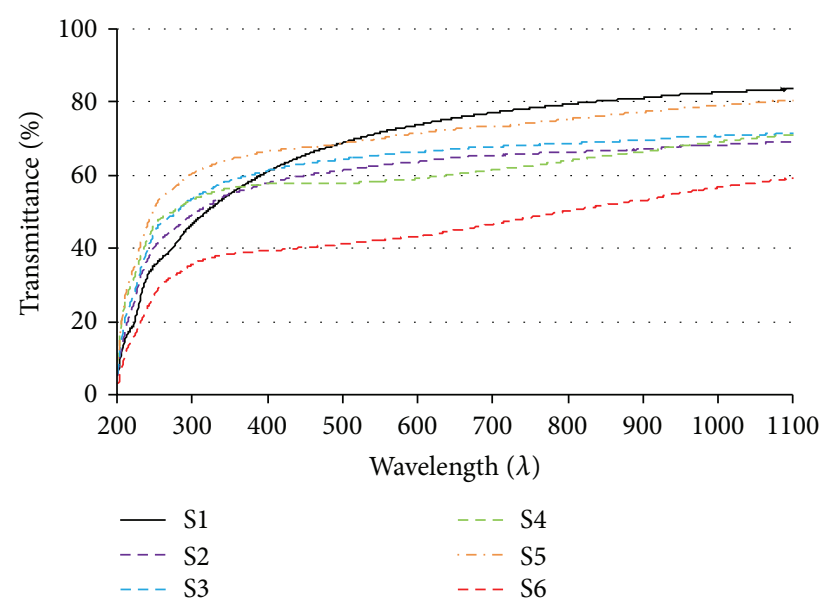

(a)

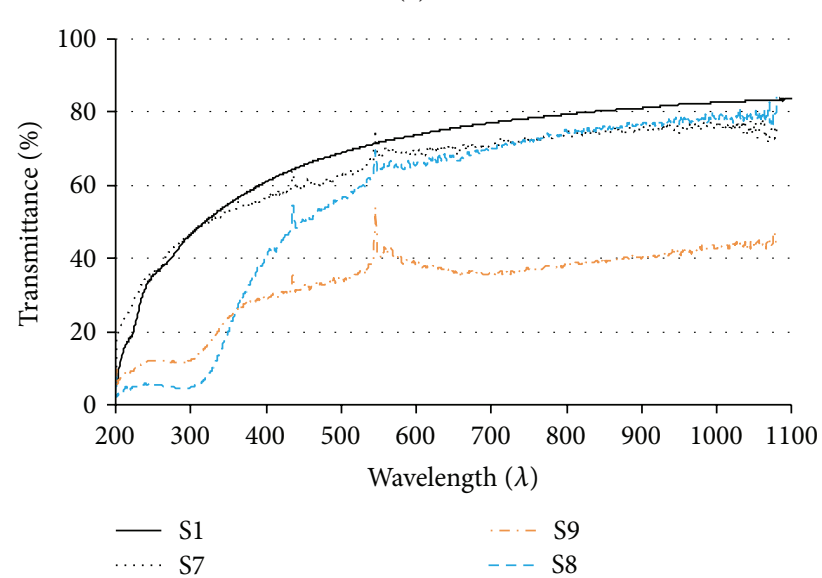

(b)

FIGURE 6: Spectral transmittance of (a) control and blend samples with NIR-reflective pigments and (b) control with NIR reflective and other UV additives.

TABLE 7: Average values in percentage for transmittance and reflectance in UV, PAR, and NIR regions for control and various blend samples.

\begin{tabular}{lcccc}
\hline Sample & $T(\%)$ UV & $T(\%)$ PAR & $T(\%)$ NIR & $R(\%)$ NIR \\
\hline S1 & 41.2 & 70.8 & 81.0 & 7.6 \\
S2 & 43.1 & 62.4 & 67.3 & 8.3 \\
S3 & 46.5 & 65.3 & 69.7 & 10.1 \\
S4 & 46.7 & 58.8 & 66.5 & 13.9 \\
S5 & 52.6 & 70.1 & 77.2 & 8.5 \\
S6 & 42.2 & 42.5 & 53.3 & 12.2 \\
S7 & 29.9 & 65.7 & 74.6 & 9.7 \\
S8 & 12.4 & 60.1 & 75.9 & 11.6 \\
S9 & 16.0 & 35.8 & 40.3 & 31.5 \\
\hline
\end{tabular}

this, a series of experiments are going on to improve the NIR reflectance by optimizing the percentage of loading and the type of NIR-reflective pigments used in the formulation.

\section{Acknowledgments}

This paper was supported by the NSTIP Strategic Technologies Program no. 09-ADV914-02 in Saudi Arabia. Special thanks are due to Mr. M. R. Shady for measuring radiometric properties of the films and to Mr. Khaja Nayeemuddin for the assistance during the preparation of formulations.

\section{References}

[1] A. M. Abdel-Ghany, I. M. Al-Helal, S. M. Alzahrani, A. A. Alsadon, I. M. Ali, and R. M. Elleithy, "Covering materials incorporating radiation-preventing techniques to meet greenhouse cooling challenges in arid regions: a review," The ScientificWorld Journal, vol. 2012, Article ID 906360, 11 pages, 2012.

[2] G. Papadakis, D. Briassoulis, G. Scarascia Mugnozza, G. Vox, P. Feuilloley, and J. A. Stoffers, "Radiometric and thermal properties of, and testing methods for, greenhouse covering materials," Journal of Agricultural Engineering Research, vol. 77, no. 1, pp. 7-38, 2000.

[3] E. Schettini and G. Vox, "Greenhouse plastic films capable of modifying the spectral distribution of solar radiation," The Journal of Agricultural Engineering, vol. 1, pp. 19-24, 2010.

[4] V. P. Sethi and S. K. Sharma, "Survey of cooling technologies for worldwide agricultural greenhouse applications," Solar Energy, vol. 81, no. 12, pp. 1447-1459, 2007.

[5] T. Kozai and S. Sase, "A simulation of natural ventilation for a multi-span greenhouse," Acta Horticulturae, vol. 87, pp. 39-49, 1978.

[6] T. Boulard and B. Draoui, "Natural ventilation of a greenhouse with continuous roof vents: measurements and data analysis," Journal of Agricultural Engineering Research, vol. 61, no. 1, pp. 27-36, 1995.

[7] T. Boulard, J. F. Meneses, M. Mermier, and G. Papadakis, "The mechanisms involved in the natural ventilation of greenhouses," Agricultural and Forest Meteorology, vol. 79, no. 1-2, pp. 61-77, 1996.

[8] A. M. Abdel-Ghany, "Energy balance model for natural ventilation of greenhouses," International Journal of Engineering Science, vol. 35, pp. 71-92, 2007.

[9] I. M. Al-Helal, "Effects of ventilation rate on the environment of a fan-pad evaporatively cooled, shaded greenhouse in extreme arid climates," Applied Engineering in Agriculture, vol. 23, no. 2, pp. 221-230, 2007.

[10] I. Al-Helal, N. Al-Abbadi, and A. Al-Ibrahim, "A study of fanpad performance for a photovoltaic powered greenhouse in Saudi Arabian summer," International Agricultural Engineering Journal, vol. 13, no. 4, pp. 113-124, 2004.

[11] G. A. Giacomelli and W. J. Roberts, "Greenhouse covering systems," HortTechnology, vol. 3, no. 1, pp. 50-58, 1993.

[12] C. Espejo, A. Arribas, F. Monzó, and P. P. Díez, "Nanocomposite films with enhanced radiometric properties for greenhouse covering applications," Journal of Plastic Film \& Sheeting, vol. 28, no. 4, pp. 336-350, 2012.

[13] G. Scarascia-Mugnozza, C. Sica, and G. Russo, "Plastic materials in European agriculture: actual use and perspectives," Journal of Agricultural Engineering, vol. 3, pp. 15-28, 2011.

[14] E. Espí, A. Salmerón, A. Fontecha, Y. García, and A. I. Real, "Plastic films for agricultural applications," Journal of Plastic Film and Sheeting, vol. 22, no. 2, pp. 85-102, 2006. 
[15] G. Vox and E. Schettini, "Evaluation of the radiometric properties of starch-based biodegradable films for crop protection," Polymer Testing, vol. 26, no. 5, pp. 639-651, 2007.

[16] A. M. Abdel-Ghany, T. Kozai, and C. Chun, "Evaluation of selected greenhouse covers for use in regions with a hot climate," Japan Journal of Tropical Agriculture, vol. 45, pp. 242-250, 2001.

[17] A. M. Abdel-Ghany, T. Kozai, and C. Chun, "Plastic films vs. fluid-roof cover for a greenhouse in a hot climate: a comparative study by simulation," Japan Journal of High Technology in Agriculture, vol. 13, pp. 237-246, 2001.

[18] I. Verlodt and P. Verschaeren, "New interference film for climate control," Acta Horticulturae, vol. 514, pp. 139-146, 2000.

[19] J. López-Marín, A. González, Y. García-Alonso et al., "Use of cool plastic films for greenhouse covering in Southern Spain," Acta Horticulturae, vol. 801, pp. 181-186, 2008.

[20] I. Impron, S. Hemming, and G. P. A. Bot, "Effects of cover properties, ventilation rate, and crop leaf area on tropical greenhouse climate," Biosystems Engineering, vol. 99, no. 4, pp. 553-564, 2008.

[21] U. N. Mutwiwa, B. Von Elsner, H. J. Tantau, and J. F. J. Max, "Cooling naturally ventilated greenhouses in the tropics by near-infra red reflection," Acta Horticulturae, vol. 801, pp. 259$265,2008$.

[22] M. K. Seo, J. R. Lee, and S. J. Park, "Crystallization kinetics and interfacial behaviors of polypropylene composites reinforced with multi-walled carbon nanotubes," Materials Science and Engineering A, vol. 404, no. 1-2, pp. 79-84, 2005.

[23] Y. Kanbur and Z. Kucukyavuz, "Electrical and mechanical properties of polypropylene/carbon black composites," Journal of Reinforced Plastics and Composities, vol. 28, no. 8, pp. 22512260, 2008.

[24] E. Lezak, Z. Kulinski, R. Masirek, E. Piorkowska, M. Pracella, and K. Gadzinowska, "Mechanical and thermal properties of green polylactide composites with natural fillers," Macromolecular Bioscience, vol. 8, no. 12, pp. 1190-1200, 2008.

[25] A. V. Shenoy, S. Chattopadhyay, and V. M. Nadkarni, "From melt flow index to rheogram," Rheologica Acta, vol. 22, no. 1, pp. 90-101, 1983.

[26] P. R. Hornsby, "Rheology, compounding and processing of filled thermoplastics," Advances in Polymer Science, vol. 139, pp. 155217, 1999.

[27] J. F. Steffe, Rheological Methods in Food Process Engineering, Freeman Press, East Lansing, Mich, USA, 2nd ed edition, 1996.

[28] H. A. Barnes, A Handbook of Elementary Rheology, University of Wales, Aberystwyth,UK, 2000. 

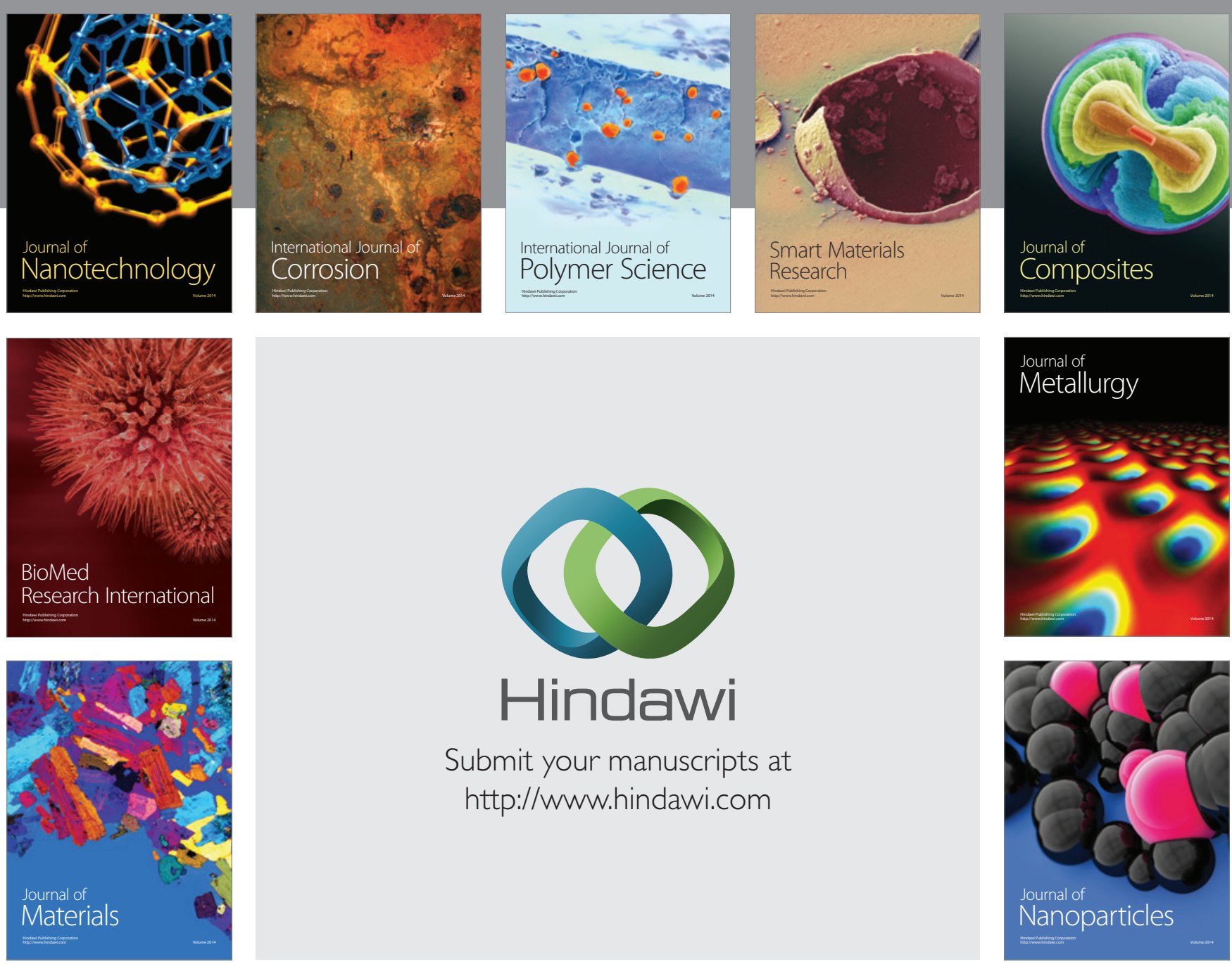

Submit your manuscripts at http://www.hindawi.com
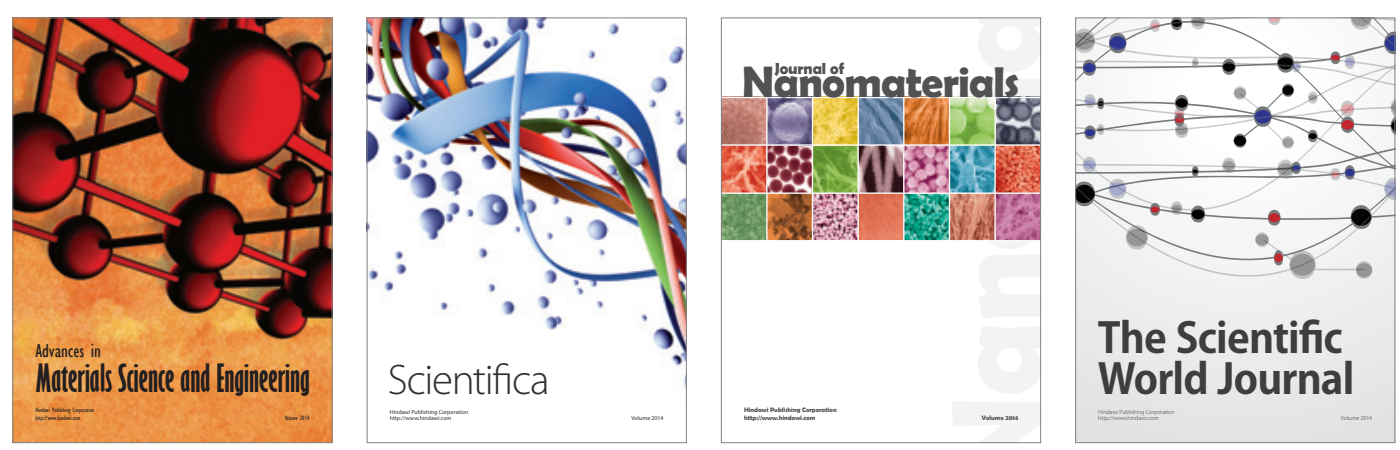

\section{The Scientific World Journal}
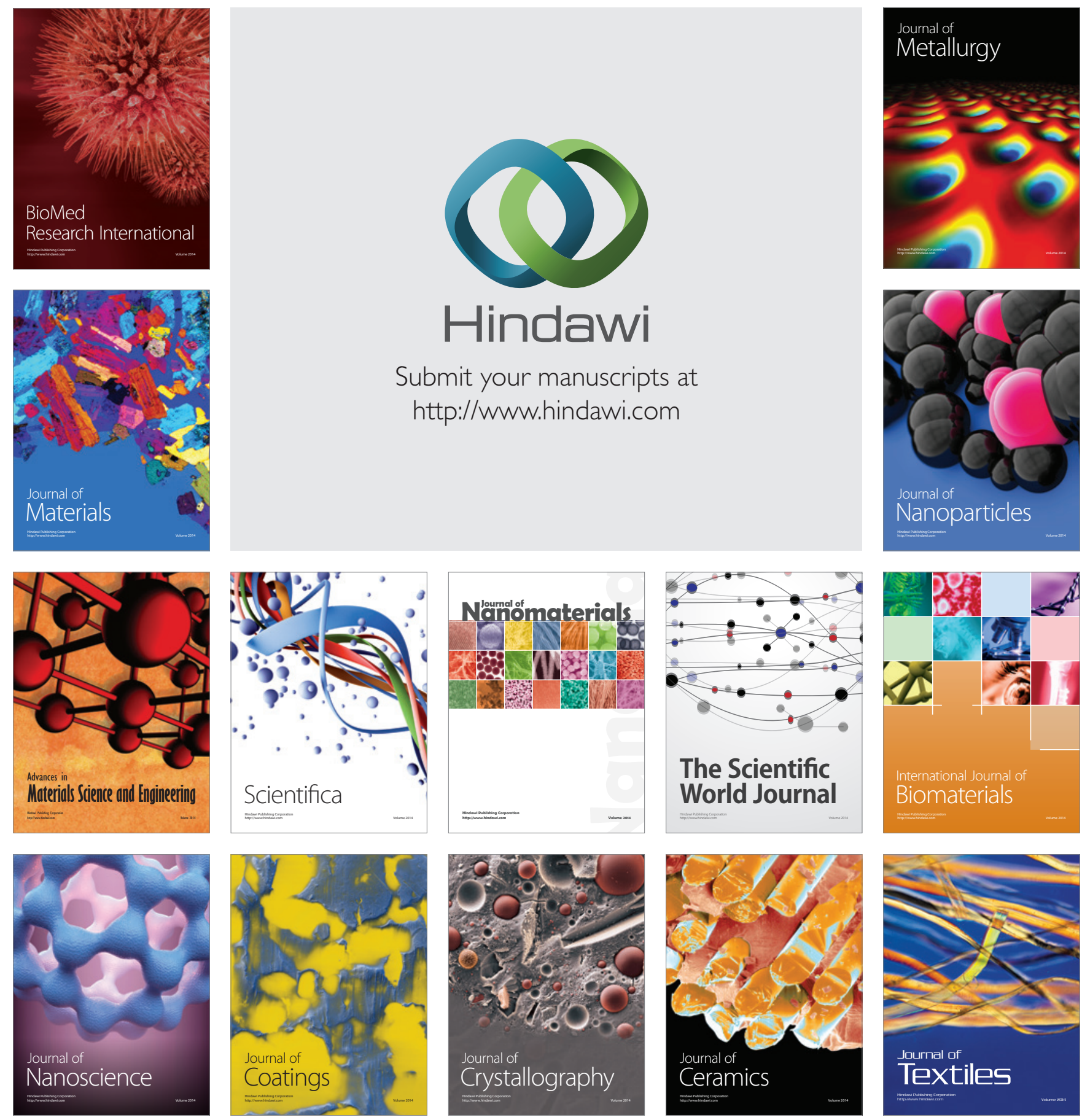